\title{
Применение Altera DSP Builder системы Matlab / Simulink для разработки имитационной модели КИХ-фильтра на параллельной распределенной арифметике
}

\author{
А. Строгонов, д. Т. н. ${ }^{1}$
}

УДК 004.4 | ВАК 05.13 .12

\begin{abstract}
Пакет расширения Altera DSP Builder системы визуально-имитационного моделирования Matlab/Simulink позволяет быстро создать имитационную модель сложного цифрового устройства, такого как КИХ-фильтр, на последовательной и параллельной распределенной арифметике с извлечением VHDL-кода. Преимущества последовательной и параллельной распределенной арифметики, реализованной в проекте на базе ПЛиС, заключаются в сокращении используемых ресурсов и повышении быстродействия . Рассмотрим пример разработки КИХ-фильтра на параллельной распределенной арифметике с использованием методологии объектно-ориентированного проектирования ЦОс-устройств с применением Altera DSP Builder системы Matlab/Simulink, сравним его характеристики с фильтром, реализованным на цОс-блоках с помощью мегафункции FIR II системы Quartus Prime.
\end{abstract}

$\mathrm{P}$ еализация КИХ-фильтров на параллельной распределенной арифметике позволяет достичь рекордного быстродействия для ПЛИс за счет использования "безумножительных" схем умножения. Причем быстродействие не снижается по мере увеличения числа отводов и коэффициентов фильтра, а также точности представления входных данных. Это актуально для проектов на базе бюджетных серий плис (особенно отечественных производителей), в которых отсутствуют аппаратные умножители или их количество ограничено, но в них представлено большое количество логических ресурсов или блочной памяти

Такой подход позволяет также отказаться от применения при разработке КИХ-фильтров различных мегафункций, использующихаппаратные умножители, встроенные в ЦОС-блоки ПлИС (ALTMULT_ADD, ALTMULT_ACCUM), например в САПР Quartus II, или мегаядер FIR Compiler либо FIR II (в Quartus Prime).

В частности, в ПЛИС российского производства серии 5576 нет встроенных умножителей, а Плис серии 5578 содержат от 14 до 66 умножителей с размерностью операндов 18 × 18. На практике требуются КИХ-фильтры с крутым

Воронежский государственный технический университет, профессор кафедры полупроводниковой электроники и наноэлектроники, тел. +7 4732 43-76-95, andreistrogonov@mail.ru. спадом АЧХ, что приводит к увеличению числа отводов до 110 и более.

ПлИс Intel серии Cyclone 10GX типа 10СX2202019 года выпуска содержат 192 ЦОС-блока с переменной точностью, количество встроенных умножителей с размерностью операндов 18×19 - 384 (или 192 для размерности 27×27), с аппаратной поддержкой формата с плавающей запятой. ПлИС CHK Intel серии Stratix типа 10DX 2019 года выпуска содержат уже 11520 умножителей с размерностью операндов $18 \times 19$

Примеры применения распределенной арифметики содержатся во всех руководствах пользователя зарубежных ПЛИС. В частности, инструмент System Generator для разработки и отладки ЦОС-систем в базисе Плис Xilinx содержит функциональный блок FIR Compiler v5.0 аналог функции FIR Compiler v5.0 генератора параметризированных ядер XLogiCORE IP [1]. В справочной

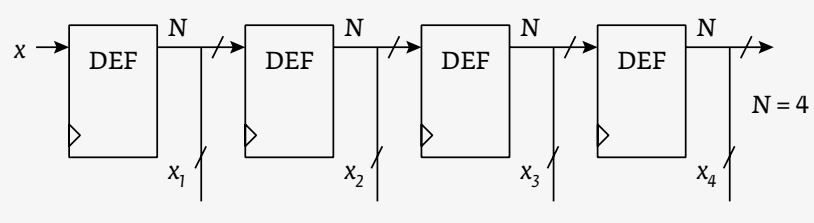

Рис. 1. Линия задержки КИХ-фильтра на регистрах 


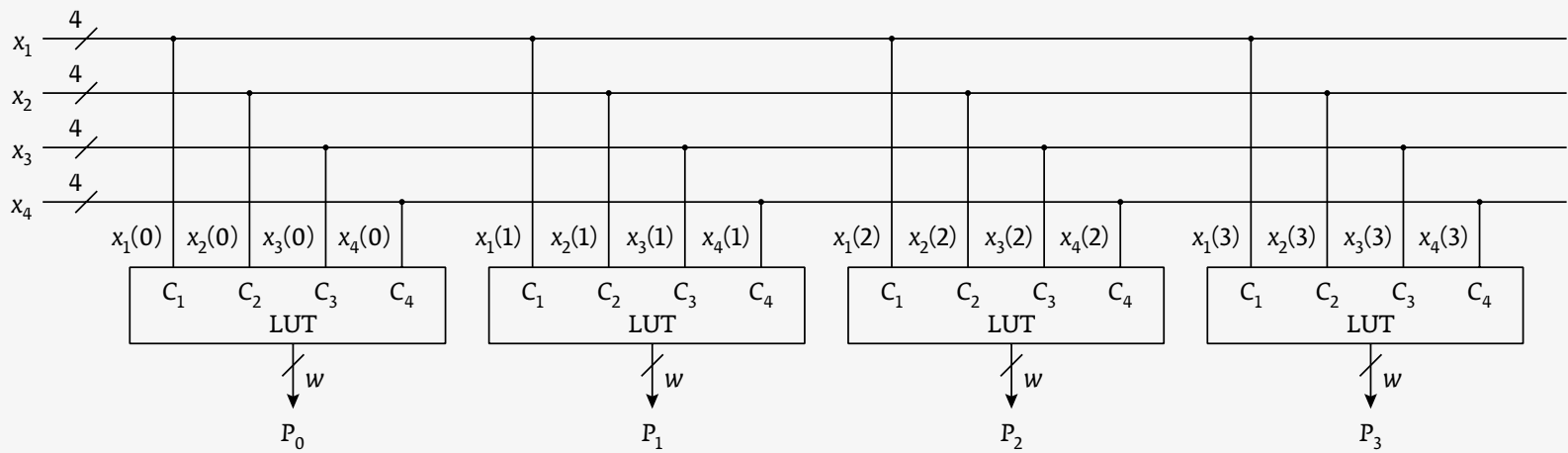

Рис. 2. Принцип подключения выходов линии задержки КИХ-фильтра на четыре отвода к 4-входовым LUT

системе пакета расширения Altera DSP Builder также можно найти примеры КИХ-фильтров на основе последовательной распределенной арифметики (по адресу: quartus\dsp_builder\DesignExamples\Demos\Filters\DA32).

Однако поскольку в современных ПЛИС содержится огромное число ЦОС-блоков, в новых версиях САПР, например от компаний Xilinх или Intel (Quartus Prime), и пакетах расширения системы Matlab/Simulink предпочтение отдается систолическим и обратнотранспонированным структурам КИХ-фильтров с использованием МАС-блоков. Чаще других применяется транспонированная

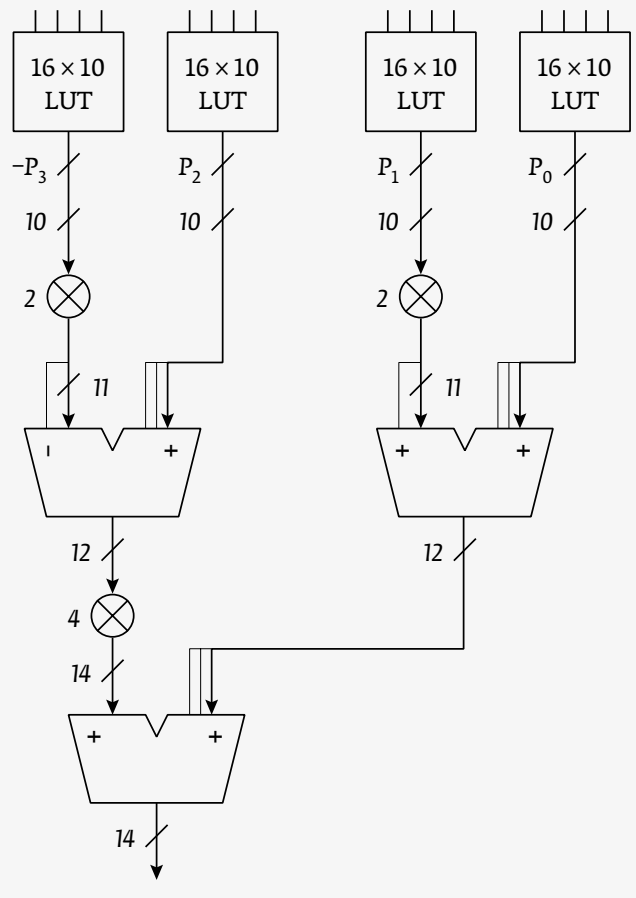

Рис. 3. Структура КИХ-фильтра на четыре отвода, четыре бита на распределенной параллельной арифметике схема, позволяющая эффективно распараллелить вычисления. Например, в САПР ПлИС Xilinx ISE14.4 функция FIR Compiler v6.3, входящая в состав генератора параметризированных ядер XLogiCORE IP, поддерживает такие структуры фильтров. Они получили название «транспонированные структуры, основанные на операциях умножения и накопления» (Transpose Multiply-Accumulate Architecture).

Инструмент System Generator от Xilinх подходит для разработки последовательных КИХ-фильтров в формате с фиксированной запятой с использованием параметризованных функциональных блоков n-tap MAC FIR Filter и n-tap Dual Port Memory MAC FIR Filter. В функциональных блоках FIR Compiler версии v6.x и старше поддержка распределенной арифметики не предусмотрена.

Покажем, что КИХ-фильтры на основе параллельной распределенной арифметики демонстрируют наивысшее быстродействие. Для этого рассмотрим пример разработки КИХ-фильтра на параллельной распределенной арифметике с использованием методологии объектно-ориентированного проектирования цифровых устройств обработки сигналов с применением пакета расширения Altera DSP Builder версии 12.1 системы визуально-имитационного моделирования Matlab / Simulink (версия 8.0.0.783 (R2012b)). Из модели извлечем VHDL-код

\section{0 ИНТЕРНЕТ-МАГАЗИН CMI H. www.SMD.ru \\ электронные компоненты для поверхностного монтажа}

НОВОЕ В ПРОГРАММЕ ПОСТАВОК

- Разборные металлические EMI SMD змраны

- Кварцевые генераторы 0532 на частоты АО 125 MГL

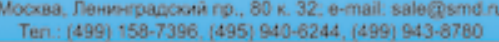


и реализуем проект в САПР Quartus II ver.13.0 (сборка 156). Для этого используем ПЛИС серии Cyclone $\mathrm{V}$ типа 5CGXFC7C7F23С8, содержащую 56480 адаптивных логических модулей (АЛМ). Сравним быстродействие фильтра при его реализации в Плис серии Cyclone V на ЦОС-блоках с помощью мегафункции FIR II CAПР Quartus Prime v.15.0.0 (сборка 145).

Уравнение КИХ-фильтра (нерекурсивного цифрового фильтра с конечно-импульсной характеристикой) представляется как арифметическая сумма произведений $[1,2]$ :

$$
y=\sum_{k=1}^{K} C_{k} \cdot x_{k}
$$

где $y$ - отклик цепи; $x_{k}-k$-я входная переменная (сигнал, подлежащий фильтрации); $C_{k}-$ весовой коэффициент k-й входной переменной (постоянный для всех n); K-число отводов фильтра. Для КИХ-фильтра на четыре отвода уравнение фильтрации будет выглядеть следующим образом: $y=C_{1} x_{1}+C_{2} x_{2}+C_{3} x_{3}+C_{4} x_{4}$. Предположим, что коэффициенты фильтра - целочисленные со знаком и равны: $C_{1}=-2, C_{2}=-1, C_{3}=7$ и $C_{4}=6$.

В случае параллельной распределенной арифметики уравнение для КИХ-фильтра на четыре отвода (разрядность 4 бита) записывается в виде [1, 2]:

$$
P_{\text {out }}=2^{0} \cdot P_{0}+2^{1} \cdot P_{1}+2^{2} \cdot P_{2}-2^{3} \cdot P_{3}
$$

где частичные произведения $\mathrm{P}_{0}, \mathrm{P}_{1}, \mathrm{P}_{2}$ и $\mathrm{P}_{3}$ равны:

$$
\begin{gathered}
P_{0}=C_{1} x_{1}(0)+C_{2} x_{2}(0)+C_{3} x_{3}(0)+C_{4} x_{4}(0)=\sum_{n=1}^{4} C_{n} x_{n}(0) ; \\
P_{1}=C_{1} x_{1}(1)+C_{2} x_{2}(1)+C_{3} x_{3}(1)+C_{4} x_{4}(1)=\sum_{n=1}^{4} C_{n} x_{n}(1) ;
\end{gathered}
$$

$$
\begin{aligned}
& P_{2}=C_{1} x_{1}(2)+C_{2} x_{2}(2)+C_{3} x_{3}(2)+C_{4} x_{4}(2)=\sum_{n=1}^{4} C_{n} x_{n}(2) \\
& P_{3}=C_{1} x_{1}(3)+C_{2} x_{2}(3)+C_{3} x_{3}(3)+C_{4} x_{4}(3)=\sum_{n=1}^{4} C_{n} x_{n}(3) .
\end{aligned}
$$

При реализации КИХ-фильтра на базе параллельной распределенной арифметики на четыре отвода необходимы четыре идентичных массива памяти, параллельно адресуемых всеми битами всех входных переменных, и свертывающее иерархическое дерево многоразрядных сумматоров, осуществляющих суммирование частичных произведений $\mathrm{P}_{0}, \mathrm{P}_{1}, \mathrm{P}_{2}$ и $\mathrm{P}_{3}$. В данном случае результат формируется за один такт, тем самым достигается наибольшее быстродействие структуры.

На рис. 1 показана линия задержки КИХ-фильтра, на рис. 2 - принцип подключения выходов линии задержки КИХ-фильтра на четыре отвода к 4-входовым LUT. Разрядность входной шины данных $N=4$. Входные данные на линии задержки представлены с 4-битной точностью параллельным кодом.

На рис. 3 представлена структура КИХ-фильтра на четыре отвода, четыре бита на распределенной параллельной арифметике. Фильтр состоит из четырех однотипных 4-входовых LUT, используемых для формирования частичных произведений $\mathrm{P}_{0}, \mathrm{P}_{1}, \mathrm{P}_{2}$ и $\mathrm{P}_{3}$ и согласно формулам (2) - (6). "Минус» перед $\mathrm{P}_{3}$ на рис. 3 означает перевод $\mathrm{P}_{3}$ в дополнительный код. 4-входовая LUT обеспечивает 16 частичных произведений, представляющих собой комбинации сумм коэффициентов фильтра с 8-битной точностью плюс два бита. Таким образом, размерность 4-входовой LUT составляет $16 \times 10$. Для суммирования значений с выходов LUT в соответствии с их весом и знаком используются два 12- и один 14-разрядный сумматоры с коррекцией разрядности на входах, чтобы исключить переполнение.

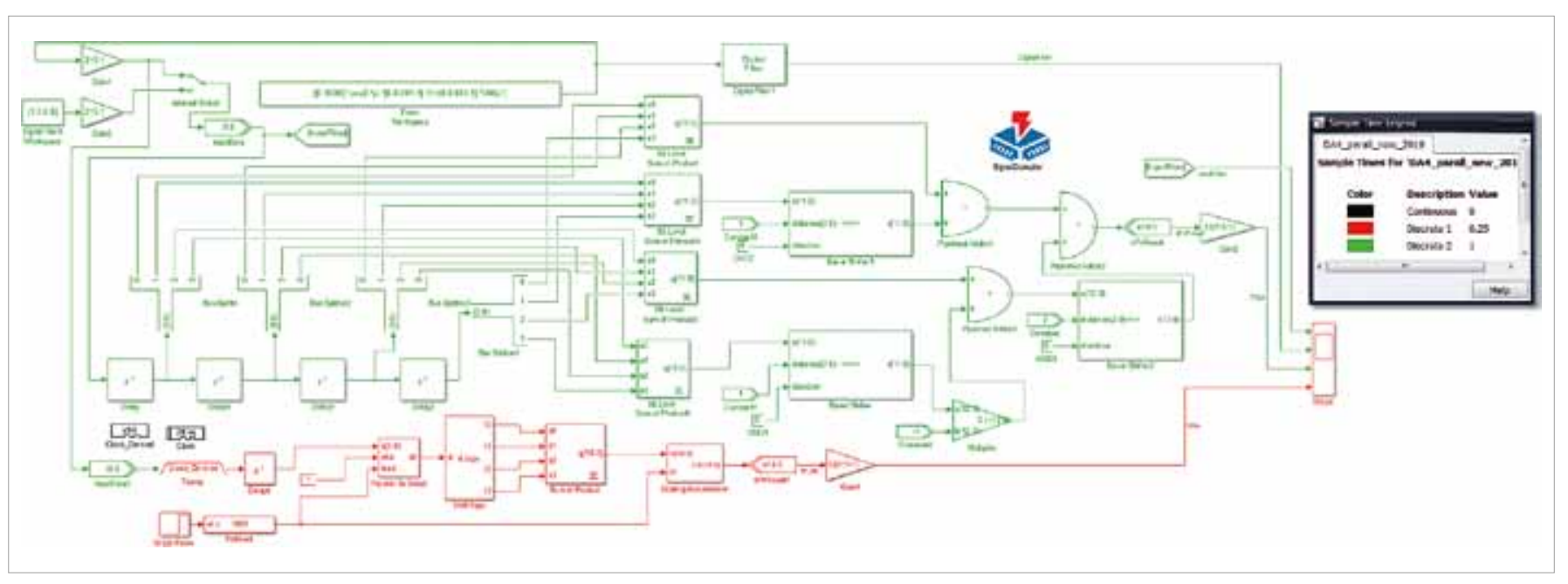

Рис. 4. Имитационные модели КИХ-фильтра на параллельной (обозначена зеленым цветом) и последовательной (обозначена красным цветом) распределенной арифметике для фильтрации лчМ-сигнала 
a)
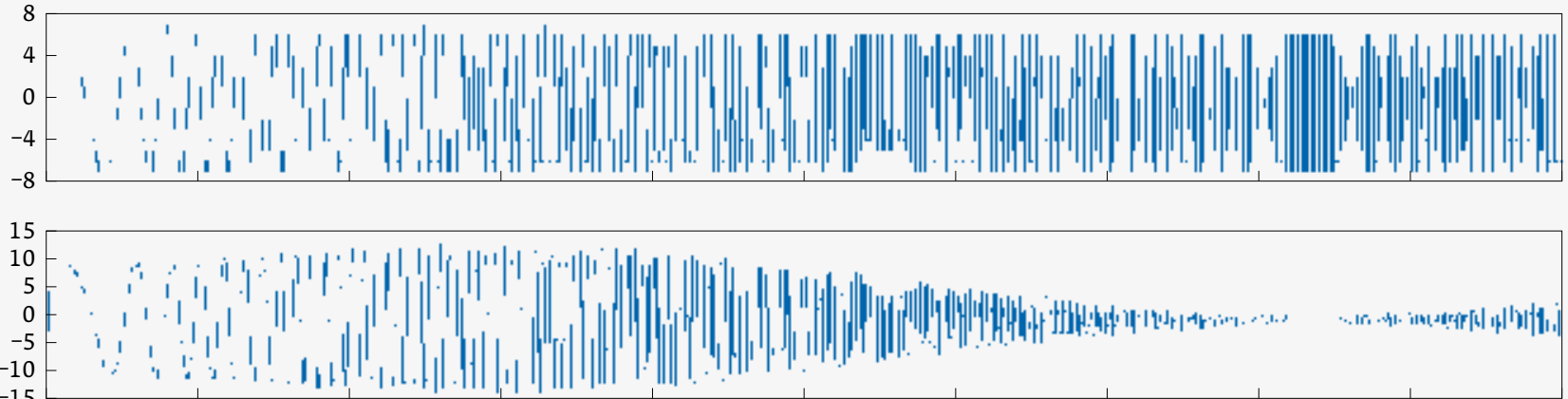

б)

$-15$

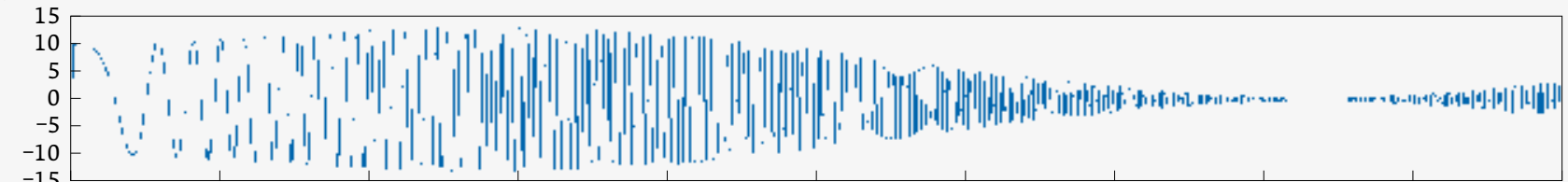

в)

$-10$

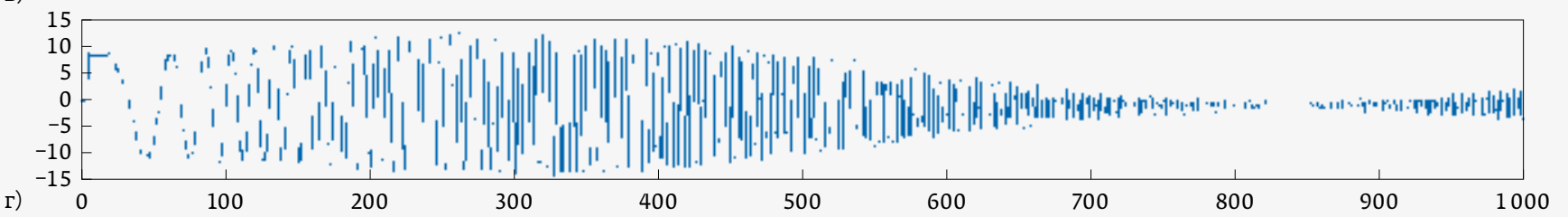

Рис. 5. Результаты имитационного моделирования фильтрации сигнала: а) ЛЧМ-сигнал; б) модель КиХ-фильтра на PDA; в) модель Digital Filter; г) модель КИХ-фильтра на SDA

На рис. 4 показаны разрабатываемая имитационная модель КИХ-фильтра на параллельной распределенной арифметике (обозначена зеленым цветом), построенная по схеме, приведенной на рис. 3, и модель КИХ-фильтра на последовательной распределенной арифметике (обозначена красным цветом) из [1].

Для краткости модель на основе параллельной арифметики назовем PDA, а на последовательной - SDA. Модели работают на разных скоростях. Для РDA-модели временной шаг симуляции в Matlab/Simulink установлен равным 1 с, а для SDA-модели - 0,25 с. Шаг симуляции для SDA-модели выбирается исходя из того, что отфильтрованные значения на выходе фильтра появляются через четыре такта синхроимпульса. За интервал дискретизации входного сигнала процесс вычисления КИХ-фильтрами на моделях PDA и SDA должен завершиться. Интервал дискретизации единичного сигнала (дельта-функция) и лчМ-сигнала (sample time) равен 1с, поэтому на схеме рис. 4 они также отмечены зеленым цветом.
Для корректной работы имитационной модели КИХ-фильтра на SDA необходимо использовать ФАПЧ для генерации дочернего синхросигнала. Для этого в модель добавлен блок Clock AlteraBlockset (Clock_Derived). В поле Base Clock Multiplicand Denomerator функционального

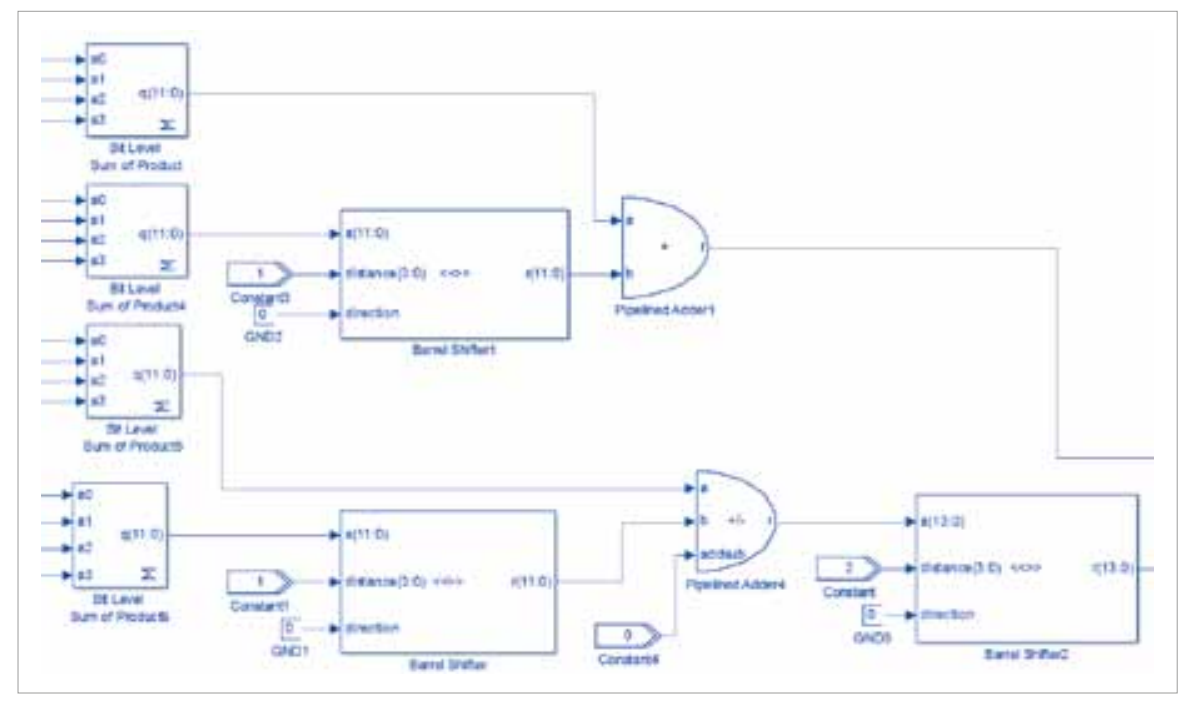

Рис. 6. Изменения в модели, позволяющие отказаться от использования умножителя для учета знака частичного произведения $P_{3}$ путем замены на сумматор/вычитатель 
Function Block Parameters: Bit Level Sum of Product BitSumProducts AlteraBlockset (mask) (link)

Performs a sum of the multiplication of one-bit inputs by signed integer fixed coefficients:

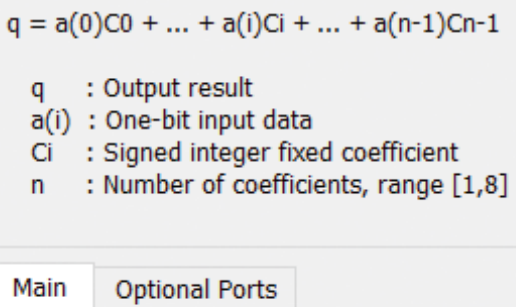

Register Inputs

\begin{tabular}{ll|l|l|l|}
\hline OK & Apply \\
\hline
\end{tabular}

\section{Рис. 7. Настройка содержимого LUT}

блока Clock AlteraBlockset подставим значение 4. С помощью функционального блока Tsamp устанавливается частота в четыре раза больше, чем у модели PDA. Имя дочернего синхросигнала: Clock_Derived.

\section{Pia Function Block Parameters: Bus Splitter4}

Bus Splitter AlteraBlockset (mask) (link)

Splits a bus into single-bit outputs.

The output ports are numbered from least significant to most significant bit. You can choose the bus type that you wish to use, and specify the number of bits on either side of the binary point.

Parameters

Bus Type Signed Integer $\checkmark$

[Number of Bits].[]

4

[].[Number of Bits]

0
Для проверки работы КИХ-фильтров на PDA и SDA, построенных с помощью пакета расширения Altera DSP Builder, дополнительно подключен функциональный блок Digital Filter (прямая форма) из библиотеки Simulink.

Входной сигнал умножается на масштабный множитель 7 (в системе Matlab: 2^3-1), отфильтрованный сигнал также делится на это значение. Масштабный множитель подбирался экспериментально, исходя из 4-разрядной точности представления входных отсчетов сигнала с учетом знака числа (в дополнительном коде 4-разрядное двоичное число перекрывает диапазон -8...+7).

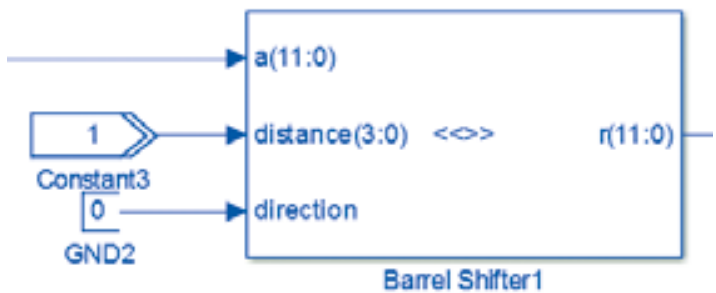

굴 Function Block Parameters: Barrel Shifter1

BarrelShifter AlteraBlockset (mask) (link)

Shifts the input data by the amount set by the distance bus. The Barrel Shifter can shift data to the left (toward the MSB) or to the right (toward the LSB).

\section{Inputs:}

a : Data input to the barrel shifter

distance : distance to shift

direction : direction to shift

Outputs:

$r$ : result after shift such as

$r=d<<$ distance when direction $=0$ (shift left)

$r=d>$ distance when direction $=1$ (shift right)

Main Optional Ports and Settings

Bus Type Signed Integer

[Number of Bits].[]

12

[].[Number of Bits]

8

$\square$ Enable Pipeline

$\checkmark$ Infer size of distance port from input port

Bit width of distance port

8

\section{OK}

Cancel

Help

Apply

Рис. 9. Функциональный блок Barrel Shifter, настроенный на сдвиг влево на один разряд 12-разрядного числа (дистанция $d=1$ ) 


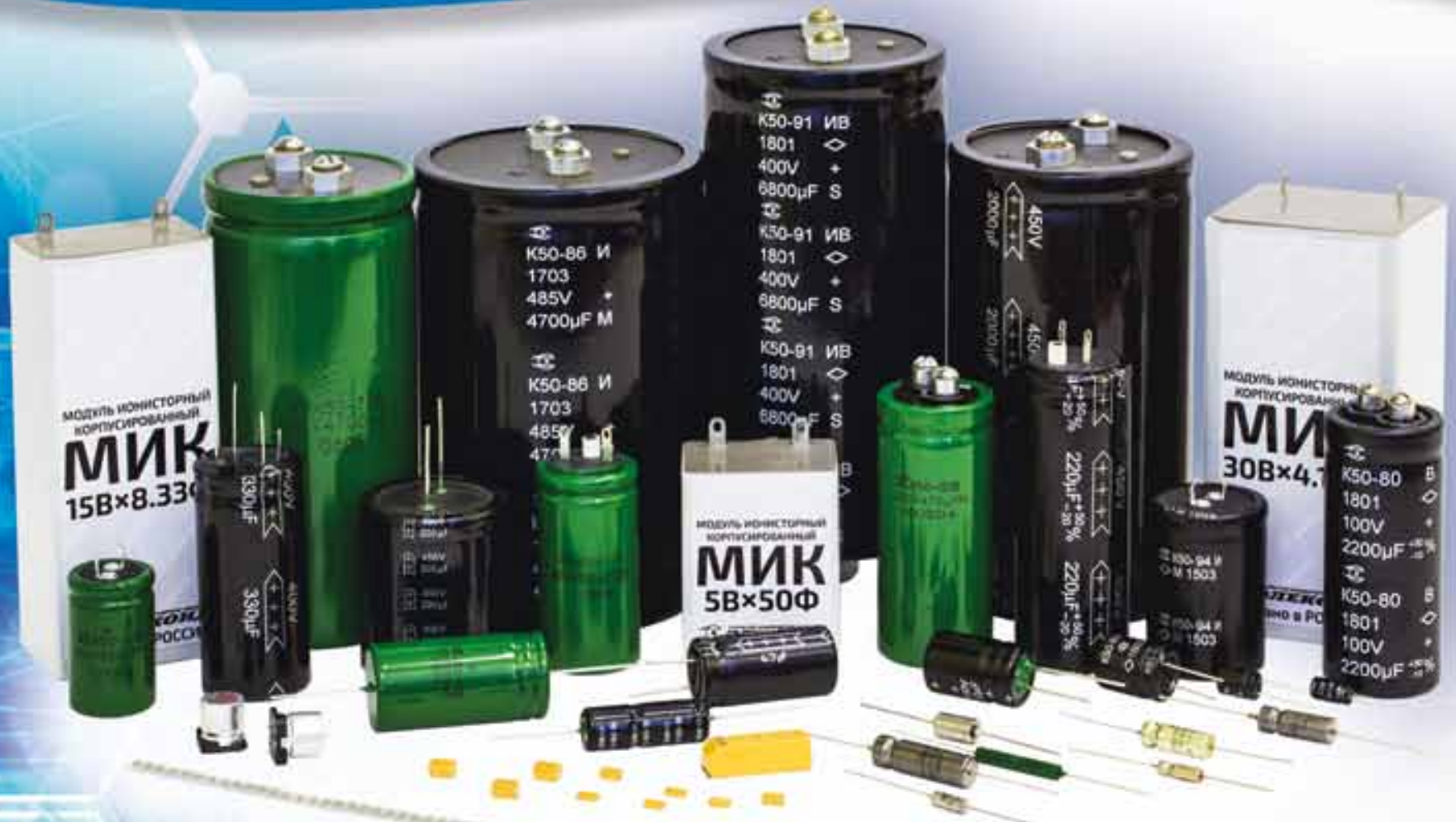

\section{Разработка и производство конденсаторов}

оксидно-электролитические алюминиевые конденсаторы К50-15, К50-17, К50-27, К50-37, К50-68, К50-77, К50-80, К50-81, К50-83, K50-84, К50-85, К50-86, К50-87, К50-88, К50-89, К50-90, К50-91, К50-92, К50-93, К50-94, К50-95(чип), К50-96, К50-97(чип), К50-98

объемно-пористые танталовые конденсаторы К52-1, К52-1М, К52-1БМ, К52-1Б, К52-9, К52-11, K52-17, К52-18, К52-19, К52-20, К52-21, К52-24, К52-26(чип), К52-27(чип), К52-28.

оксидно-полупроводниковые танталовые конденсаторы К53-1А, К53-7, К53-65(чип), К53-66, К53-68(чип), К53-69(чип), К53-71(чип), К53-72(чип), К53-74(чип), К53-77(чип), К53-78(чип), К53-82

суперконденсаторы (ионисторы) К58-26, К58-27

накопители электрической энергии на основе модульной сборки суперконденсаторов

Система менеджмента качества сертифицирована на соответствие требованиям ISO 9001

Россия, 427968, Удмуртская Республика, г. Сарапул, ул. Калинина, 3 тел.: (34147) 2-99-53, 2-99-89, 2-99-77, факс: (34147) 4-32-48, 4-27-53 e-mail: elecond-market@elcudm.ru, http://www.elecond.ru 
Для модели КИХ-фильтра на PDA коэффициенты не масштабируются, а для КИХ-фильтра на SDA применяется специальная техника масштабирования, подробно рассмотренная в $[3,4]$.

Модель состоит из линии задержки на функциональных блоках Delay, служащих регистрами, блоков Bus Splitter разделяющих 4-разрядные отводы фильтра на битовые значения, генераторов булевых функций (LUT), трех устройств сдвига данных Barrel Shifter влево на один (2 шт.) и на два разряда (что равносильно умножению на 2 и 4

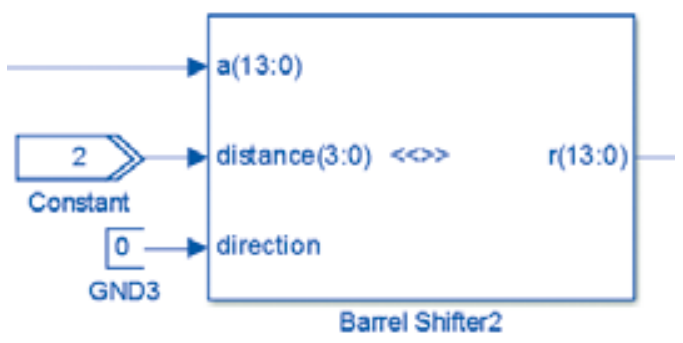

\section{듬 Function Block Parameters: Barrel Shifter2}

BarrelShifter AlteraBlockset (mask) (link)

Shifts the input data by the amount set by the distance bus. The Barrel Shifter can shift data to the left (toward the MSB) or to the right

(toward the LSB).

Inputs:

a : Data input to the barrel shifter

distance : distance to shift

direction : direction to shift

Outputs:

$r$ : result after shift such as

$r=d<<$ distance when direction $=0$ (shift left)

$r=d>$ distance when direction $=1$ (shift right)

Main Optional Ports and Settings

Bus Type Signed Integer

[Number of Bits].[]

14

[].[Number of Bits]

8

$\square$ Enable Pipeline

$\checkmark$ Infer size of distance port from input port

Bit width of distance port

8

\section{OK}

Cancel

Help

Apply

Рис. 10. Функциональный блок Barrel Shifter, настроенный на сдвиг влево на два разряда 14-разрядного числа (дистанция $d=2$ )
Таблица 1. Оценка быстродействия КИХ-фильтра на четыре отвода, реализованного в базисе ПлиС серии Cyclone V типа 5CCXFC7C7F23С8 (временная модель slow $1100 \mathrm{mV} 85 \mathrm{C}$ )

\begin{tabular}{|c|c|c|c|}
\hline $\begin{array}{l}\text { Оцениваемый } \\
\text { параметр }\end{array}$ & $\begin{array}{c}\text { КИХ- } \\
\text { фильтр } \\
\text { на SDA }\end{array}$ & $\begin{array}{c}\text { КиХ- } \\
\text { фильтр } \\
\text { на PDA }\end{array}$ & $\begin{array}{c}\text { КИХ-фильтр } \\
\text { на ЦОС-блоках } \\
\text { (мегаядро FIR II) }\end{array}$ \\
\hline $\begin{array}{l}\text { Максималь- } \\
\text { ная рабочая } \\
\text { частота, МГц }\end{array}$ & 226,45 & 407,83 & 254,39 \\
\hline $\begin{array}{l}\text { Рабочая } \\
\text { частота } \\
\text { в наихудшем } \\
\text { случае, МГц }\end{array}$ & 180,02 & 407,83 & 220,02 \\
\hline
\end{tabular}

соответственно), а также умножителя для учета знака числа. На рис. 5 показаны результаты имитационного моделирования фильтрации сигнала: лчМ-сигнал; модель КИХ-фильтра на PDA; модель Digital Filter из библиотеки Simulink; модель KИХ-фильтра на SDA. Совпадение результатов фильтрации подтверждает правильность работы имитационных моделей.

В дальнейшем от умножителя на константу -1 необходимо отказаться, так как при синтезе VHDL-кода будет использоваться умножитель ЦОс-блока. На рис. 6 показаны изменения в модели, которые необходимо внести, чтобы отказаться от применения умножителя для учета знака частичного произведения путем его замены на сумматор/вычитатель. Такая замена не влияет на логику работы устройства.

Битовые сигналы с выходов разделителей Bus Splitter подключены на основе правила: все младшие разряды всех четырех отводов подключены к 4-входовой LUT (Bit Level Sum of Product), формирующей частичное произведение $\mathrm{P}_{0}$; все старшие разряды всех четырех отводов подключены к 4-входовой LUT, формирующей частичное произведение $\mathrm{P}_{1}$, и так далее, пока не сформируется частичное произведение $\mathrm{P}_{3}$.

Рассмотрим блок BaseClock. Для функционального моделирования в Altera-ModelSim задаются синхросигнал с периодом 20 нс (поле Real-World Clock Period) и временной шаг симуляции в Matlab/Simulink, равный 1 с (поле Simulink Sample Time).

На рис. 7 показано окно настройки содержимого LUT. Коэффициенты представлены с 10-разрядной точностью, а суммы коэффициентов с 12-разрядной. Для такого фильтра требуются четыре идентичные LUT.

Линия задержки проектируемого фильтра построена на регистрах из функциональных блоков Delay. Число уровней конвейеризации блока - один. На рис. 8 представлены 


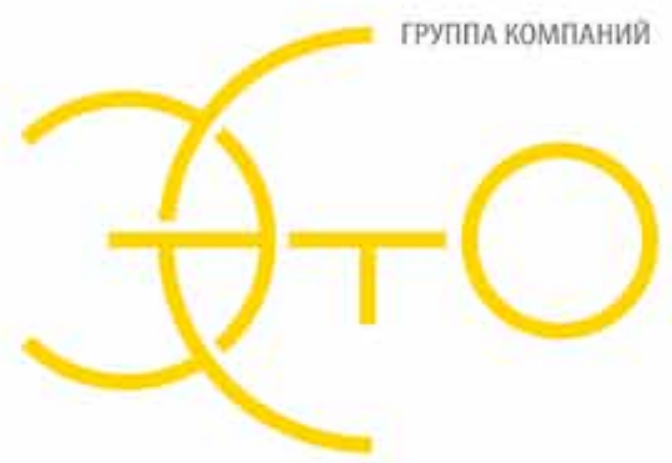

ЭЛЕКТРОННОЕ СПЕЦИАЛЬНОЕ ТЕХНОЛОГИЧЕСКОЕ ОБОРУДОВАНИЕ
Акционерное общество

«Научно-производственное предприятие

щлектронное специальное

технологическое оборудованиеж

124460, г. Москва, Зеленоград, Георгиевский проспект, А.5. стр.1 тел: (499) 729-7751, факс: (499) 479-1239 info@nppesto.ru www.nppesto.ru

CUCTEMA GROOVY ICP ТЕХНОЛОГИЯ БУДУЩЕГО Для критического травления диэлектриков на основе уникального узкозазорного индуктивного плазменного реактора для массового производства

Paзработка AO анпП बСТО, при финансовой $>$ поддержке Министерства промышленности. и торговли Российской Федерации

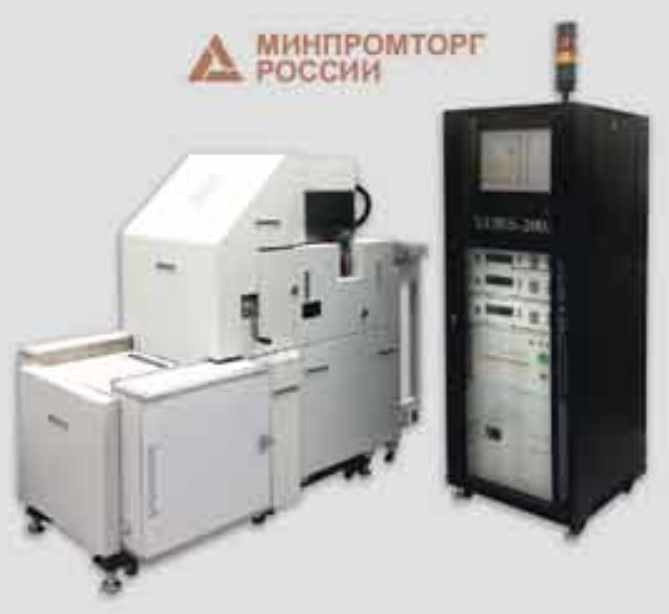

Приглашаем посетить наш стенд на выставке «ElectronTechExpo 2020", которая пройдет с 11 по 13 августа в МВЦ «Крокус Экспо»
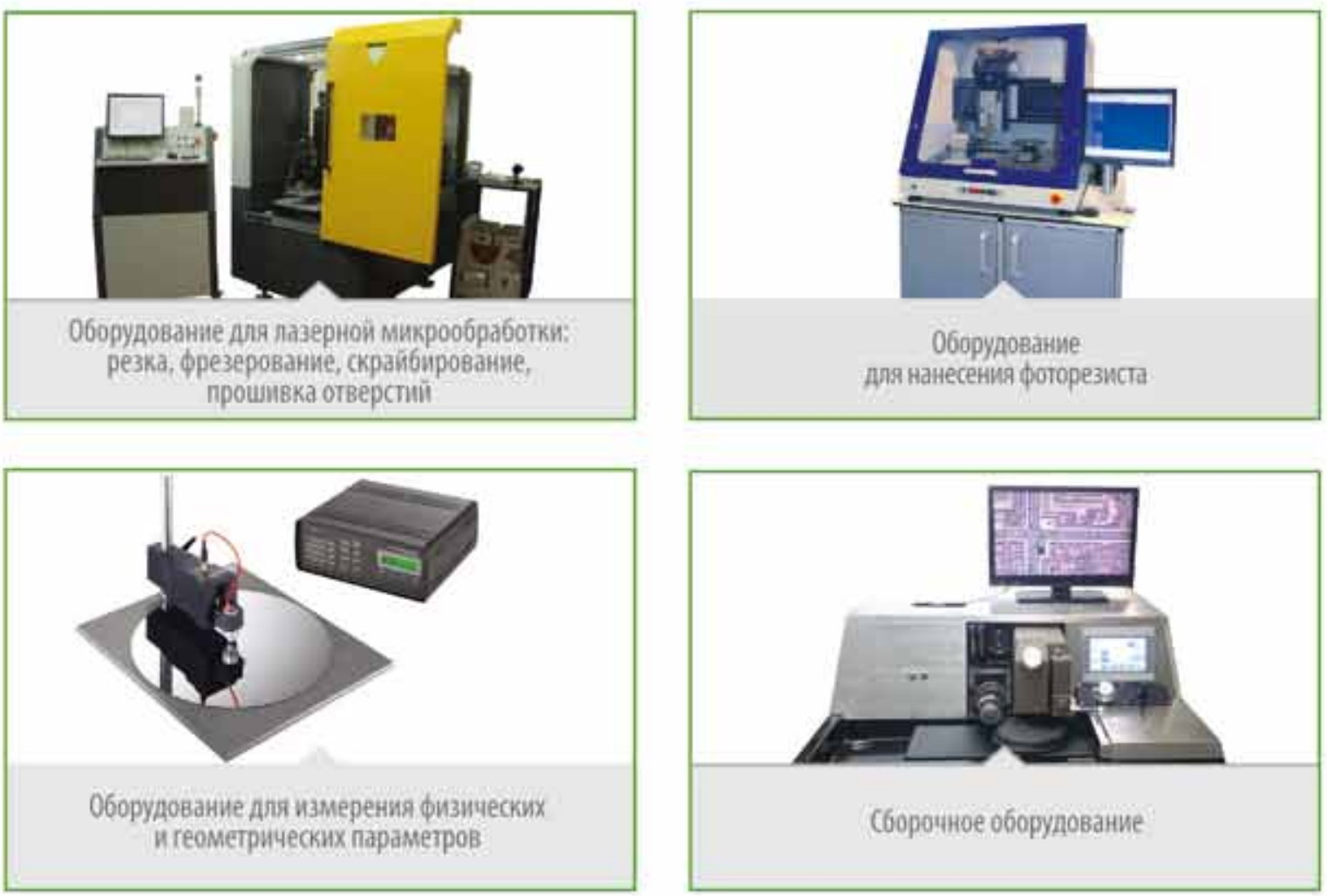


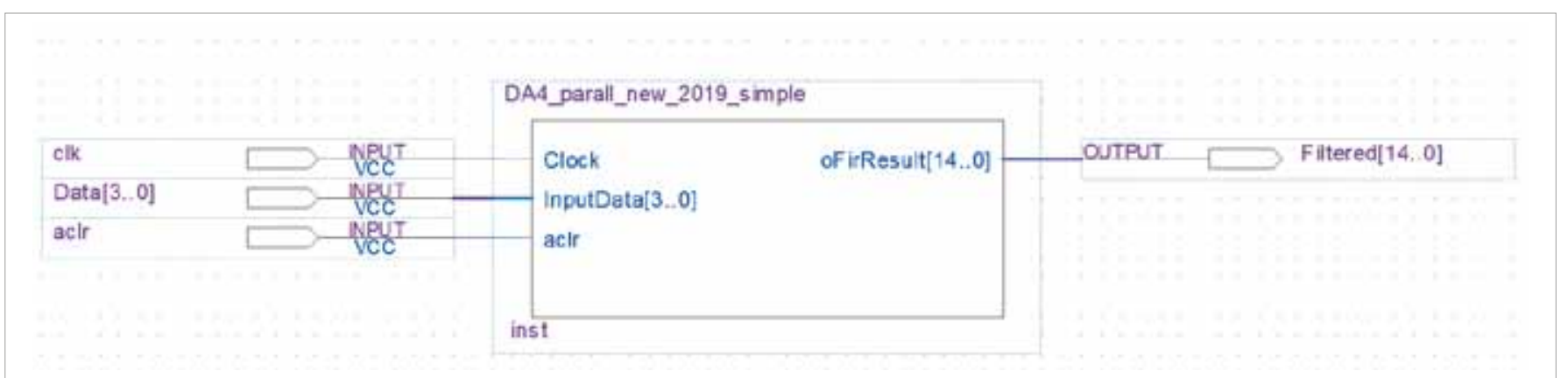

Рис. 11. Проект КИХ-фильтра, реализованный в CAПР Quartus II по VHDL-коду, извлеченному в автоматическом режиме из имитационной модели

настройки функционального блока Bus Splitter (шинный разделитель), разделяющего 4-разрядную шину на биты. Таких блоков в модели четыре.

На рис. 9 показаны настройки 12-разрядного устройства сдвига данных влево на один разряд, что равносильно умножению на два. Направление сдвига определяется портом direction. Дистанция сдвига задается портом distance $(d=1)$. На рис. 10 представлены настройки 14-разрядного устройства сдвига влево на два разряда, что равносильно умножению на четыре. Суммирование результатов сдвига выполняет дерево сумматоров, показанное на рис. 4.

Из имитационной модели КИХ-фильтра на PDA (рис. 6) извлечем VHDL-код с помощью функционального блока SignalCompiler и реализуем проект в САПР Quartus II ver.13.0 (рис. 11)

\begin{tabular}{|c|c|c|}
\hline \multicolumn{3}{|c|}{ Analysis \& Synthesis Resource Usage Summary } \\
\hline & Resource & Usage \\
\hline 1 & Estimate of Logic utilization (ALMs needed) & 33 \\
\hline \multicolumn{3}{|l|}{2} \\
\hline 3 & $\Delta$ Combinational ALUT usage for logic & 59 \\
\hline 1 & -7 input functions & 0 \\
\hline 2 & -6 input functions & 0 \\
\hline 3 & -5 input functions & 0 \\
\hline 4 & -4 input functions & 16 \\
\hline 5 & $-<=3$ input functions & 43 \\
\hline \multicolumn{3}{|l|}{4} \\
\hline 5 & Dedicated logic registers & 16 \\
\hline \multicolumn{3}{|l|}{6} \\
\hline 7 & I/O pins & 21 \\
\hline 8 & Total DSP Blocks & 0 \\
\hline 9 & Maximum fan-out node & clk input \\
\hline 10 & Maximum fan-out & 16 \\
\hline 11 & Total fan-out & 264 \\
\hline 12 & Average fan-out & 2.26 \\
\hline
\end{tabular}

На рис. 12 представлена оценка задействованных ресурсов на этапе анализа и синтеза в САПР при реализации проекта в базис плИС серии Cyclone V типа 5CGXFC7C7F23C8 (содержит 156 ЦОС-блоков). Для проекта КИХ-фильтра на PDA требуются 33 АЛМ, из них адаптивных LUT в режиме 4-входовых LUT - 16, в режиме меньше или равно 3 LUT - 43, выделенных регистров АЛМ - 16, ЦОС- блоков 0. Архитектурное планирование кристалла Плис серии Cyclone $V$ типа 5CGXFC7C7F23C8 показывает, что в проекте задействуются логические ресурсы (АЛМ) и блочная память типа M10К.

Разработаем проект в САПР Quartus Prime v.15.0 (рис. 13) с использованием мегаядра FIR II (altera_fir_compiler_ii), позволяющего быстро создавать КИХ-фильтры прямой формы с использованием

\begin{tabular}{|l|c|l|}
\hline & \multicolumn{1}{|c|}{ Resource } & \multicolumn{1}{c|}{ Usage } \\
\hline 2 & Estimate of Logic utilization (ALMs needed) & 22 \\
\hline 3 & Combinational ALUT usage for logic & 13 \\
\hline 1 & -7 input functions & 0 \\
\hline 2 & -6 input functions & 0 \\
\hline 3 & -5 input functions & 0 \\
\hline 4 & -4 input functions & 0 \\
\hline 5 & $-<=3$ input functions & 13 \\
\hline 4 & & \\
\hline 5 & Dedicated logic registers & 43 \\
\hline 6 & & \\
\hline 7 & I/O pins & 22 \\
\hline 8 & & 2 \\
\hline 9 & Total DSP Blocks & \\
\hline 10 & & $\mathrm{ck}$ input \\
\hline 11 & Maximum fan-out node & 45 \\
\hline 12 & Maximum fan-out & 228 \\
\hline 13 & Total fan-out & 2.24 \\
\hline 14 & Average fan-out & \\
\hline & & \\
\hline
\end{tabular}

Рис. 12. Оценка задействованных ресурсов при реализации КИХ-фильтра на четыре отвода в базис ПлиС серии Cyclone V типа 5CGXFC7C7F23С8: слева - PDA в САПР Quartus II; справа - мегаядро FIR II CAПP Quartus Prime 


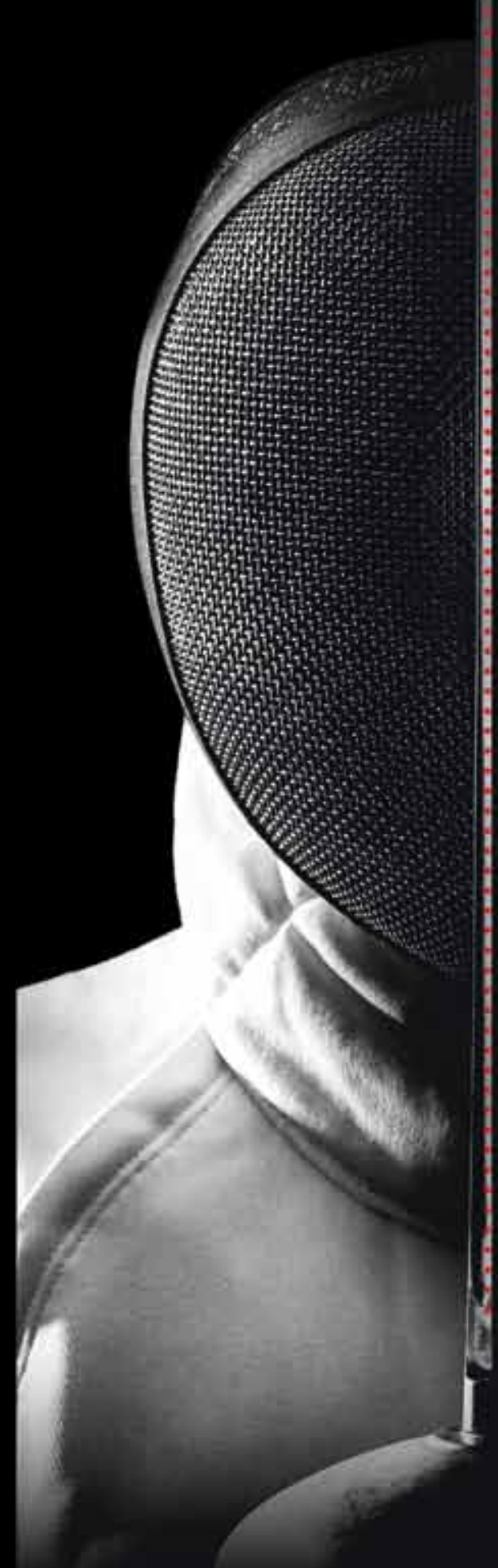

\section{pic sun \\ AGILE ALD}

\section{Для компаний, нацеленных на инновации}

Компания Picosun является лидирующим поставщиком технологии AGILE ALD® (атомно-слоевое осаждение, ACO) для микроэлектроники и других областей промышленности.

Спектр оборудования PICOSUN@ варьируется от полностью автоматизированного до совместимых с SEMI 300-MM промышленных систем АСО. Оборудование предназначено для проведения НИОКР и небольших пилотных производств с низкой стоимостью владения. Picosun предлагает системы "Под ключ" для производств с использованием пластин до 200 мм и для обработки 3D-компонент.

Picosun предлагает лучшие в своем классе технологии осаждения тонких пленок для производства традиционных ИС, составных и силовых полупроводников, светодиодов, МЭМС, датчиков и 3D-Компонент. K последним относатся медицинские приборы и имплантаты, сборки печатных плат, различные детали машин, часов, монеты и украшения.

Решения для нанесения покрытий от Picosun: превосходное качество процессов, гибкий и инновационный дизайн оборудования, различные уровни послепродажной поддержки и технического обслуживания.

www.picosun.ru

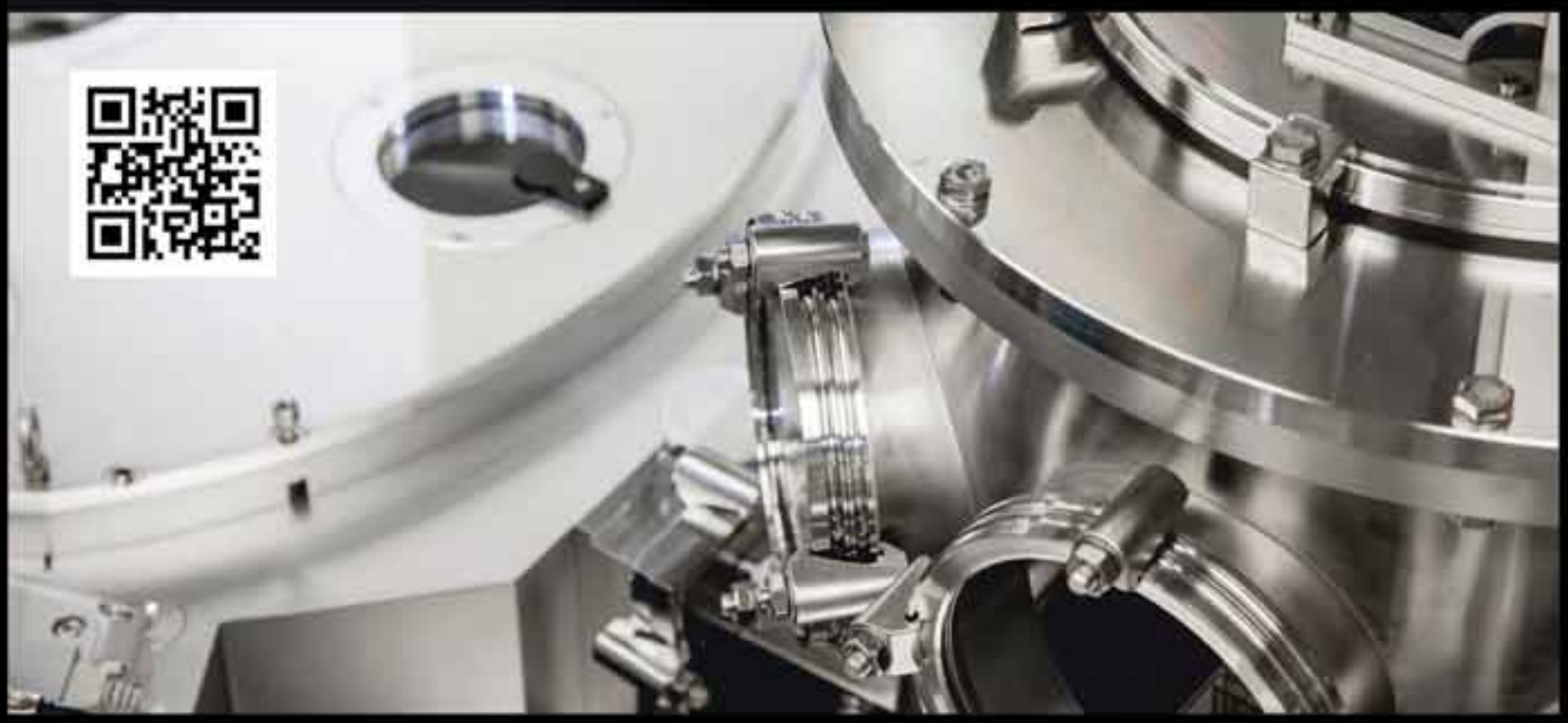




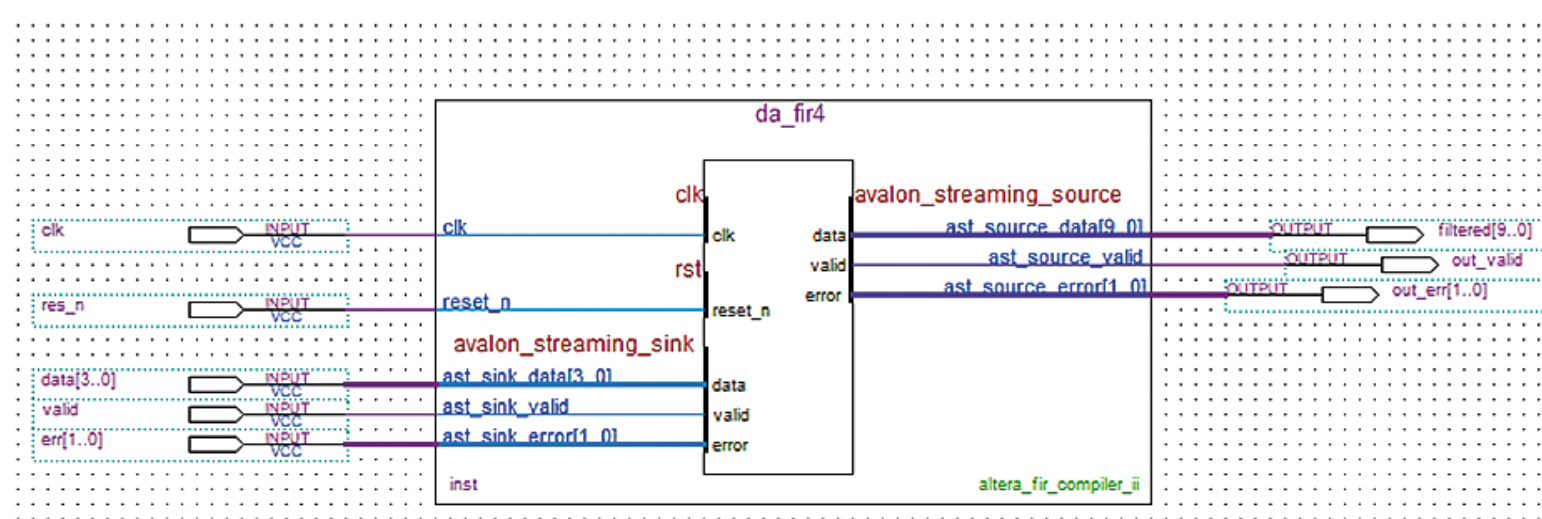

Рис. 13. Проект КИХ-фильтра с использованием мегаядра FIR II в CAПP Quartus Prime v.15.0

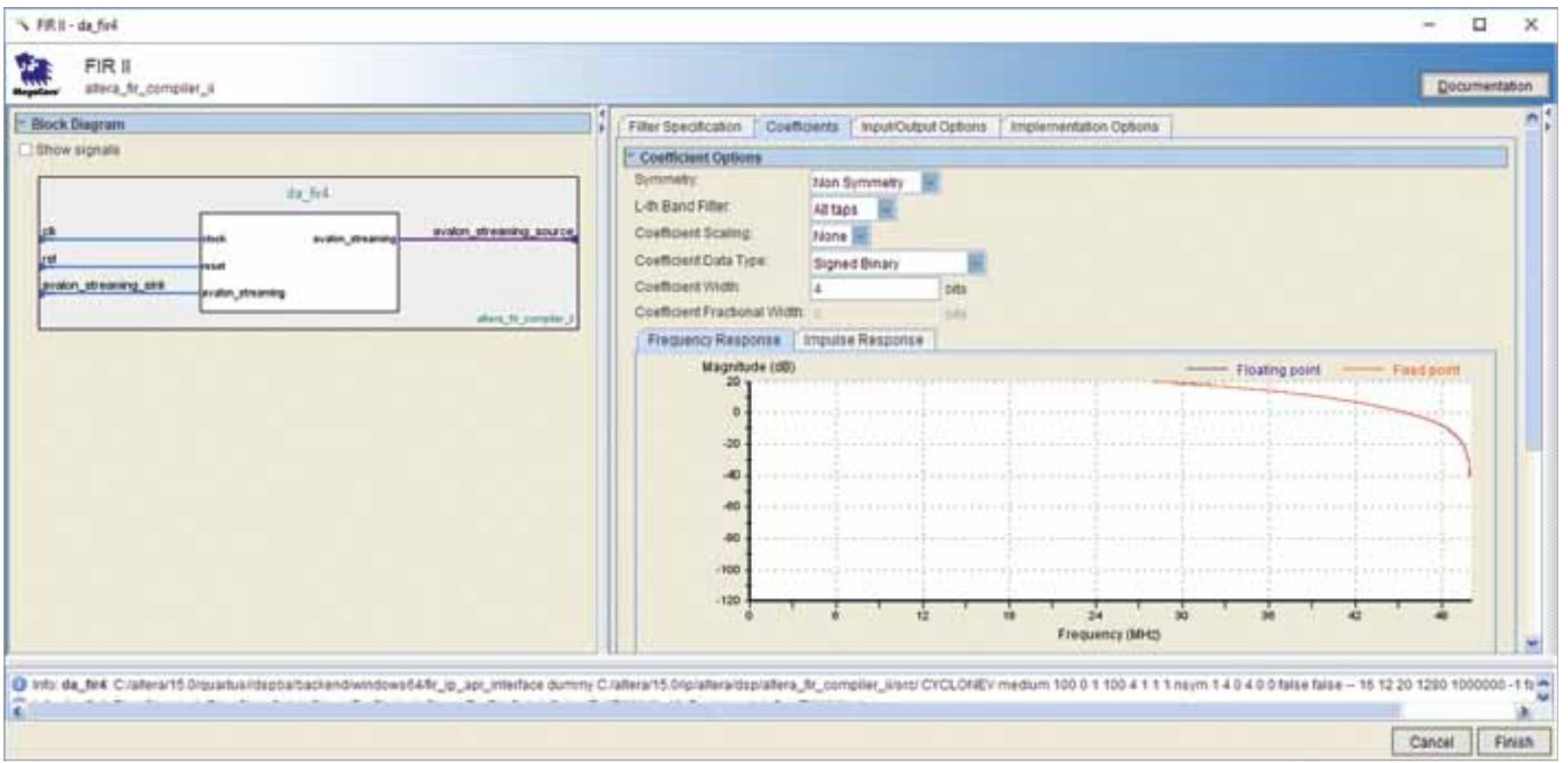

Рис. 14. Настройки мегаядра FIR II CAПР Quartus Prime v.15.0 (АЧХ фильтра)

\section{0 смп}

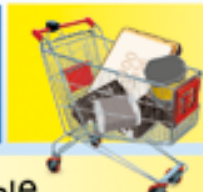

ИНТЕРНЕТ-МАГАЗИН www.SMD.ru

электронные для поверхностного монтажа

HOBOE В ПРОГРАMME ПОСТАВОК

- Катушим индуктивности на токи до $10 \mathrm{~A}$

- U.FL pazbevы и pigtail co SMA

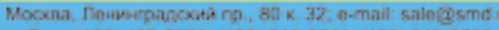

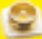

аппаратных умножителей в ЦОс-блоках. Коэффициенты фильтра -2, -1, 7 и 6 загрузим из предварительно созданного текстового файла в формате .txt. Peaлизуем проект в такой же плис серии Cyclone $\mathrm{V}$ типа 5CGXFC7C7F23C8. На рис. 14 показаны настройки мегаядpa FIR II CAПP Quartus Prime v.15.0 (АЧХ фильтра, закладка "коэффициенты»). На рис. 15 показано архитектурное планирование кристалла плис серии Cyclone $\vee$ типа 5CGXFC7C7F23С8. Задействуются АЛМ и два ЦОС-блока (четыре умножителя)

Оценка ресурсов, задействованных на этапе анализа и синтеза при реализации проекта в базис плис 


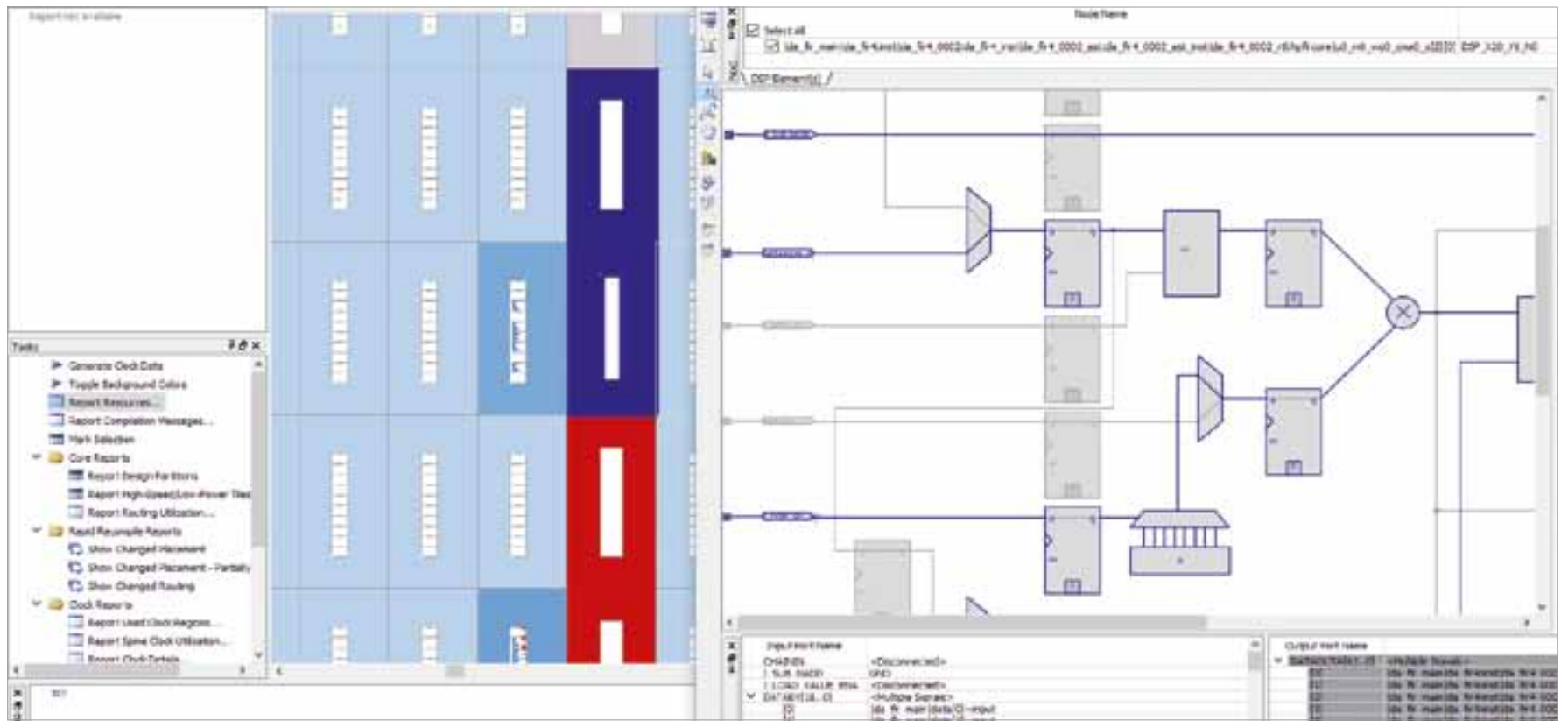

Рис. 15. Архитектурное планирование кристалла ПлИС серии Cyclone V типа 5CGXFC7C7F23C8

серии Cyclone V типа 5CGXFC7C7F23C8 с использованием мегаядра FIR II, показывает, что для реализации проекта требуются 22 АЛМ, из них адаптивных LUT для комбинационной логики в режиме меньше или равно трем LUT - 13, выделенных регистров АЛM для последовательностной логики - 43 и ЦОС-блоков - 2 (см. рис. 12)

В табл.1 приведена оценка максимальной рабочей частоты и рабочей частоты в наихудшем случае для функциональных моделей КИХ-фильтра на SDA, PDA и ЦОС-блоках (мегаядро FIR II) при использовании медленной временной модели в условиях критического напряжения питания ядра Плис 1,1 В и температуры $85^{\circ} \mathrm{C}$ (модель slow 1100 mV 85C), полученных с помощью TimeQuest. Анализ представленных в таблице данных показывает, что возможен двукратный выигрыш по быстродействию в случае использования КИХ-фильтра на PDA.

\section{ЛИТЕРАТУРА}

1. Реализация алгоритмов цифровой обработки сигналов в базисе программируемых логических интегральных схем: Учебное пособие / 4-е изд., испр. и доп. - СПб: Изд-во "Лань", 2019. 352 с.

2. Строгонов А., Быстрицкий А. КИХ-фильтр на распределенной арифметике: проектируем сами // Компоненты и технологии. 2013. № 3. С. 131-138.

3. Строгонов А., Городков П. Проектирование цифровых устройств обработки сигналов в системе визуальноимитационного моделирования Matlab/Simulink с использованием Altera DSP Builder // Компоненты и технологии. 2018. № 12. C. 22-27.

4. Строгонов А., Городков П. Проектирование КИХ-фильтра на распределенной арифметике в системе визуальноимитационного моделирования Matlab/Simulink с использованием Altera DSP Builder // Компоненты и технологии. 2019. № 2. C. 88-94.

\section{0 «АК Микротех»}

Комплексные решения в области микрозлектронного и микросборочно̆ого производства

Поставка, наладка и ремонт технологического оборудования:

Отработка и постановка технолотическй процессов

обеспечение материалами и комплектующими

WWW.AKMICROTECH.RU +7(499) 3980770 SALES@AKMICROTECH.RU

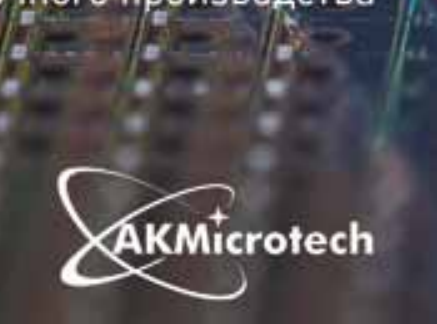


